\title{
Adverse events during immunotherapy in Slovenian patients with metastatic melanoma reveal a positive correlation with better treatment outcomes
}

\author{
Tanja Mesti1 ${ }^{1}$ Vid Ceplak Mencin ${ }^{1}$, Biljana Mileva Boshkoska ${ }^{3,4}$, Janja Ocvirk ${ }^{1,2}$ \\ ${ }^{1}$ Department of Medical Oncology, Institute of Oncology Ljubljana, Ljubljana, Slovenia \\ ${ }^{2}$ Faculty of Medicine, University of Ljubljana, Ljubljana \\ ${ }^{3}$ Faculty of information studies in Novo mesto, Novo mesto, Slovenia \\ ${ }^{4}$ Department for Knowledge Technologies, Institute Jožef Stefan, Ljubljana Slovenia
}

Radiol Oncol 2021; 55(3): 354-361.

Received 24 November 2020

Accepted 16 March 2021

Correspondence to: Prof. Janja Ocvirk, M.D., Ph.D., Institute of Oncology Ljubljana, Zaloška 2, Ljubljana. E-mail: jocvirk@onko-i.si

Disclosure: No potential conflicts of interest were disclosed.

This is an open access article under the CC BY-NC-ND license (http://creativecommons.org/licenses/by-nc-nd/4.0/).

Background. Immunotherapy with CTLA-4 inhibitors and PDI checkpoint inhibitors has initiated a breakthrough in the treatment and prognosis of patients with metastatic melanoma. The survival of these patients has increased from the expected survival time of less than 12 months to at least forty months. However, immunotherapy with either antiCTLA-4 antibodies or PD1 inhibitors alone or in combination has a broad palette of significant immune-related adverse events. The aim of the study was to assess the correlation of immune-related adverse events with treatment outcomes defined as significant differences in the overall response rate (ORR) and progression-free survival (PFS) of patients, who developed immune-related adverse events during immunotherapy.

Patients and methods. A retrospective analysis of patients with metastatic melanoma treated with immunotherapy in 2020 at the Oncology Institute of Ljubljana was performed. Only patients with radiological evaluation of the immunotherapy response were included. The patients were divided into two cohorts: a cohort of patients with immune-related adverse events (irAE group) and a cohort of patients with no immune-related adverse events (NirAE group). Significantly better overall response and progression-free survival in the irAE cohort defined the primary aim of our study. To investigate the differences in progression-free survival between the irAE cohort and NirAE cohort, we used survival analysis. In particular, a Cox proportional hazards model with covariates of time to progression and adverse events was used for survival analysis. The Kruskal-Wallis $\mathrm{H}$-test was applied, and a $\mathrm{p}$-value of $\mathrm{p}<=0.05$ was considered the cut-off point for a statistically significant difference between the groups.

Results. Among the 120 patients treated with immunotherapy, radiological response evaluation was performed for 99 patients: 38 patients in the irAE cohort and 61 patients in the NirAE cohort. The ORRs for the irAE and NirAE cohorts were $57 \%$ and $37 \%$, respectively. The PFS was significantly better for the irAE cohort (301.6 days) than for the NirAE cohort (247.29 days). The results of the survival regression analysis showed a significant increase in the survival probability from less than $60 \%$ for the NirAE cohort to almost $80 \%$ for the irAE cohort.

Conclusions. Patients with metastatic melanoma treated with immunotherapy who developed immune-related adverse events showed better treatment outcomes with longer times to disease progression and better overall response rates than patients treated with immunotherapy who did not develop immune-related adverse events, with a significant increase in the survival probability from less than $60 \%$ for the NirAE cohort to almost $80 \%$ for the irAE cohort.

Key words: immune related adverse events; immunotherapy; melanoma; metastases; response; survival 


\section{Introduction}

Ipilimumab, an anti-CTLA-4 antibody, was the first immunotherapy approved for the treatment of metastatic malignant melanoma and is associated with a median 5-year overall survival rate of 20 months. $^{1}$ Significantly longer response times were achieved with the checkpoint PD1 inhibitors pembrolizumab and nivolumab, with a 5-year overall survival rate of approximately 40 months. ${ }^{1-3}$ Ipilimumab in combination with nivolumab results in an extension of the overall survival time to 60 months. ${ }^{1}$

Immunotherapy with either anti-CTLA-4 antibodies or PD1 inhibitors alone or in combination has a broad spectrum of significant immunologically related adverse events, such as immunologically related skin toxicity, pneumonitis, thyroid dysfunction and other endocrinopathies, hepatitis, and renal dysfunction. ${ }^{1-3}$

At the Institute of Oncology Ljubljana, a national centre for the treatment of patients with metastatic melanoma, we used immunotherapy on a daily basis. The PD1 inhibitors pembrolizumab and nivolumab are the main inhibitors used, as well as anti-CTLA-4 antibodies in combination with nivolumab, in accordance with the Slovenian national guidelines, based on the European Society for Medical Oncology (ESMO) and National Comprehensive Cancer Network (NCCN) guidelines for the treatment of metastatic melanoma. ${ }^{4-6}$ The past years of work with patients on immunotherapy have led us to the unusual observation that patients who experience immune-related adverse events have a better treatment outcome in terms of time to relapse. Several recent studies from different melanoma centres and one meta-analysis showed that regardless of the cancer type, irAEs exhibited a positive correlation with ORR, PFS and OS.-9 The meta-analysis revealed that the ORR of irAE patients with melanoma was $37.67 \%$ but was $23.44 \%$ in NirAE patients. PFS and OS were significantly longer in the irAE population. In particular, the PFS for irAE ranged from 17.61 months to unreached and for NirAE ranged from 2.23 to 3 months. The OS for irAEs and NirAEs was 15.24 months and 8.94 months, respectively. ${ }^{9}$ Hence, the aim of this study was to assess the correlation of immune-related adverse events and treatment outcomes defined as significant differences in the overall response rate (ORR) and progression-free survival (PFS) of patients who developed immunerelated adverse events during immunotherapy treatment.

\section{Patients and methods}

A retrospective analysis of patients with metastatic melanoma treated with immunotherapy from January to July 2020 was performed at the Institute of Oncology Ljubljana. Data were collected from the clinical database. The study included only metastatic melanoma patients with radiographic evaluations of immunotherapy treatment. The iRECIST (immune Response Evaluation Criteria in Solid Tumours) criteria were used to evaluate the tumour response. Patient characteristics, including age, sex, Eastern Cooperative Oncology Group (ECOG) performance status, systemic treatment prior to immunotherapy, stage of melanoma, histology type and location of primary melanoma, were recorded. The patients were divided into two cohorts: the cohort of patients with immunerelated adverse events (irAE group) and the cohort of patients without immune-related adverse events (NirAE). The irAEs were evaluated by a clinician based on the findings of laboratory tests, clinical examinations, and imaging studies. The irAEs (with a potential immunologic cause) were graded according to the National Cancer Institute Common Terminology Criteria for Adverse Events, version 4.0.

The Python programming language was used for statistical calculations. The Kruskal-Wallis H-test was applied, and a p-value of $p<=0.05$ was considered the threshold for statistical significance.

For survival analysis, we used the Cox proportional hazards model with the covariates time to progression and adverse events. For each patient, we considered the length of time from introduction of immunotherapy to the time the study analysis was performed. We considered whether the patient remained alive throughout the study duration and the occurrence of an adverse event. The hazard rate was assumed to be a Weibull distribution. Posterior survival probabilities were obtained by Monte Carlo simulation implemented in Python using the package pymc3.

The study was approved by the Institutional Review Board Committee and was carried out according to the Declaration of Helsinki.

\section{Results}

From January to July 2020, 120 patients with metastatic melanoma were treated with immunotherapy. Seventy-six patients did not develop immunerelated adverse events, and 44 patients developed 
TABLE 1. Baseline characteristics of the cohorts

\begin{tabular}{|c|c|c|c|}
\hline Characteristics & & $\begin{array}{l}\text { irAE cohort } \\
\text { n (\%) }\end{array}$ & $\begin{array}{l}\text { NirAE cohort } \\
\text { n (\%) }\end{array}$ \\
\hline Number & & $38(38)$ & $61(62)$ \\
\hline Age mean & & 67.4 & 61.6 \\
\hline \multirow[t]{2}{*}{ Sex } & Male & $18(47.4)$ & $37(60.7)$ \\
\hline & Female & $20(52.6)$ & $24(39.3)$ \\
\hline \multirow[t]{2}{*}{ Treatment } & Naive & $34(89.5)$ & $51(83.6)$ \\
\hline & Previously treated & $4(10.5)$ & $10(16.4)$ \\
\hline \multirow[t]{3}{*}{ Immunotherapy } & Pembrolizumab & $34(89.5)$ & $52(85.2)$ \\
\hline & Nivolumab & $2(5.3)$ & $5(8.2)$ \\
\hline & Nivolumab + ipilimumab & $2(5.3)$ & $4(6.6)$ \\
\hline \multirow[t]{3}{*}{ BRAF status } & BRAF mutated & $10(26.3)$ & $17(27.9)$ \\
\hline & BRAF wild type & $21(55.3)$ & $27(44.3)$ \\
\hline & Not reported & $7(18.4)$ & $17(27.9)$ \\
\hline$M 1 a / b$ & Cohort $a$ and $b$ & $22(57.9)$ & $35(57.4)$ \\
\hline $\mathrm{Mlc} / \mathrm{d}$ & Cohort c and d & $16(42.1)$ & $26(42.6)$ \\
\hline LDH & increased & $7(18.4)$ & $15(24.6)$ \\
\hline LDH & normal & 31 (81.6) & $46(75.4)$ \\
\hline
\end{tabular}

irAE cohort = patients with metastatic melanoma who developed immune-related side effects during immunotherapy; $\mathrm{LDH}=$ lactate dehydrogenase; $\mathrm{Mla} / \mathrm{b}=$ Distant metastasis to skin, soft tissue including muscle and/or nonregional lymph node and lungs; $\mathrm{Mlc} / \mathrm{d}=$ Distant metastasis to other visceral sites and to the central nervous system (CNS); NirAE cohort = patients with metastatic melanoma who did not develop immune-related side effects during immunotherapy

immune-related adverse events. Radiological evaluation (PET CT or CT) of the immunotherapy treatment response was performed for 99 out of 120 patients who were included in our study. The included patients were divided into two cohorts. The cohort of immunotherapy-treated patients who did not develop immune-related adverse events (NirAE cohort) included $61(61,62 \%)$ patients, and

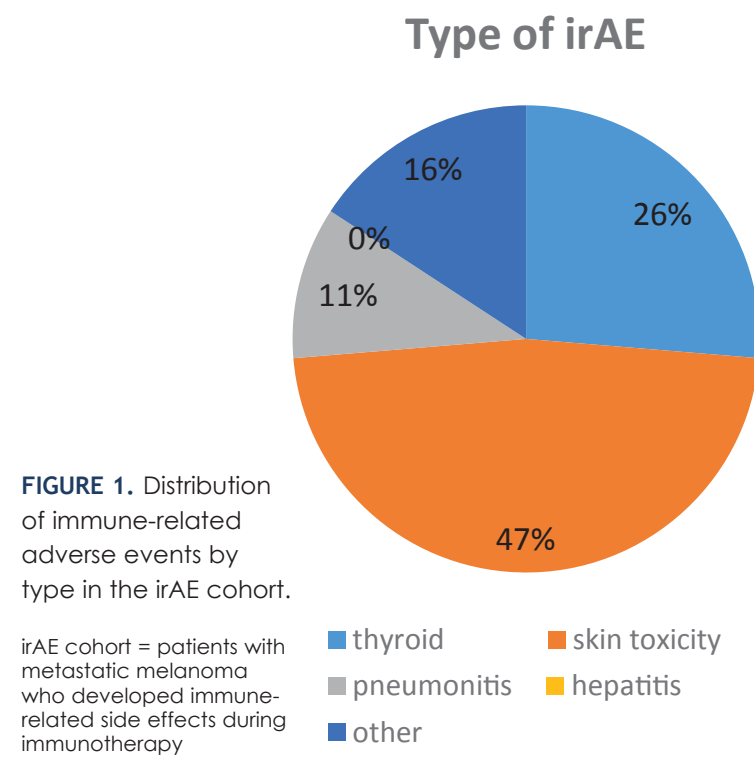

the cohort of patients who developed immunerelated side effects (irAE cohort) included 38 (38, $38 \%$ ) patients. The baseline characteristics of both cohorts are presented in Table 1.

Of the 38 patients in the irAE cohort, 10 patients had thyroiditis (hyperthyroiditis/hypothyroiditis), 18 patients had skin toxicity (vitiligo, rash, itchy skin, dermatitis), 4 had pneumonitis, none had hepatitis, and 6 had other adverse events (arthritis, colitis, fatigue). The distribution of the immune-related adverse events of the immunotherapy in the irAE cohort is presented in Figure 1 below.

Most of the immune-related adverse events were grade 1 or 2 . One patient developed grade 3 immune-related adverse events in the form of psoriasiform dermatitis, and immunotherapy had to be discontinued. One patient with colitis presented with diarrhoea, and four patients with pulmonary toxicity presented with pneumonitis (Figure 2). No immune-related adverse events of grade 4 or 5 were present.

The radiological response evaluation was performed for 99 patients, 61 patients representing the NirAE cohort and 38 patients representing the irAE cohort. The overall response rates (ORRs) for the irAE and NirAE cohorts were $57 \%$ and $37 \%$, respectively. Complete response was achieved in $14 \%$ of patients in the irAE cohort and in $4 \%$ of patients in the NirAE cohort. The irAE cohort had a higher rate of partial response $(44 \%)$ than the NirAE cohort (34\%). One-third (31\%) of the NirAE cohort had progressive disease, and only $14 \%$ of

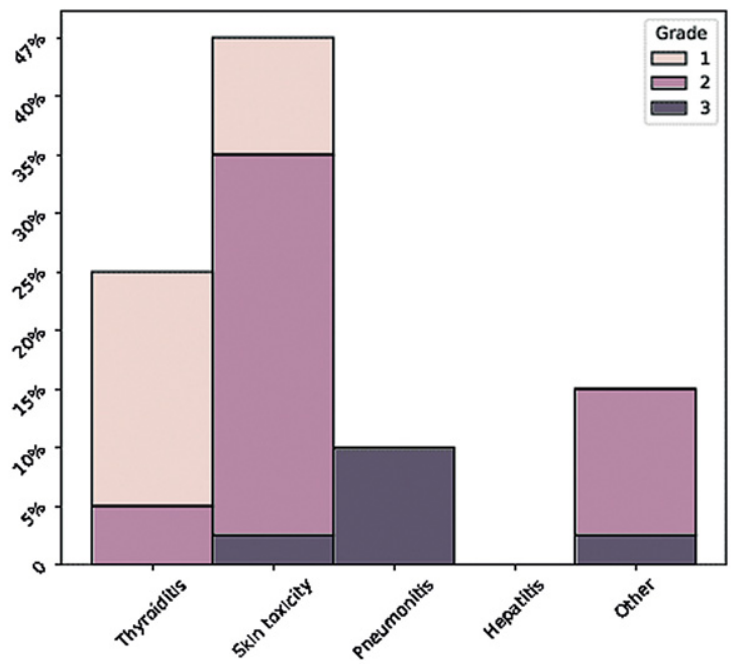

FIGURE 2. Distribution of immune-related adverse events by grade (1-3) regarding the type of immune-related adverse event in the irAE cohort presented as a percentage (\%).

irAE cohort = patients with metastatic melanoma who developed immune-related side effects during immunotherapy 


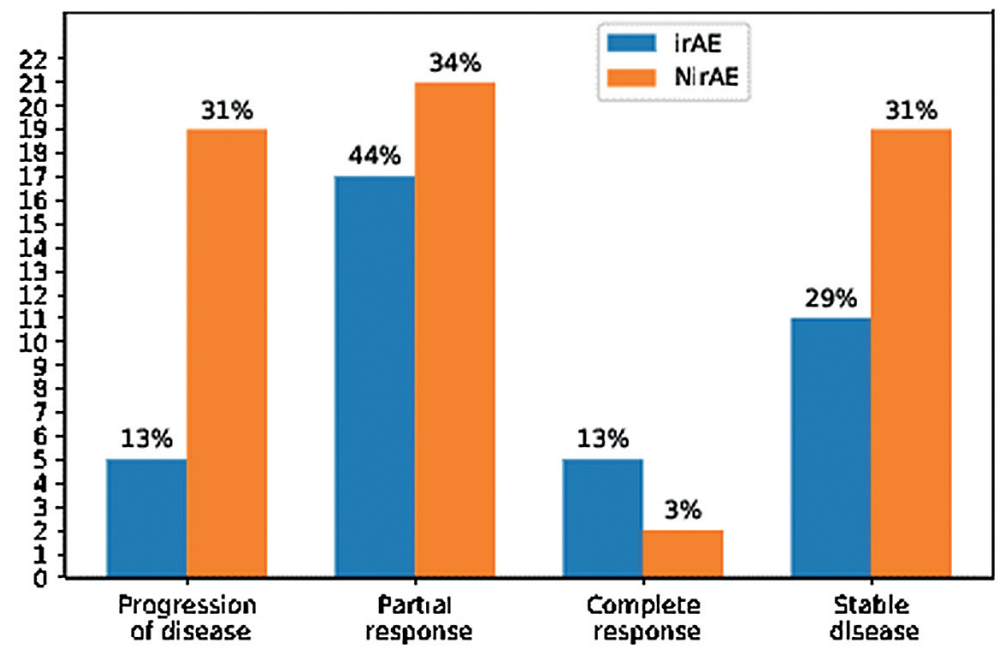

FIGURE 3. Distribution of the treatment response between the irAE and NirAE. The numbers above the bars represent the percentages with respect to its cohort, while the bar height is the absolute number of patients and is given on the $Y$ axis.

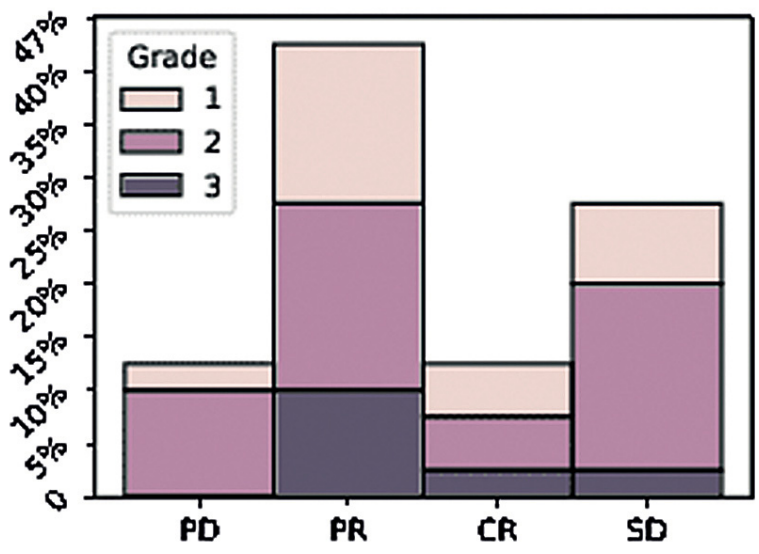

FIGURE 4. Correlation between the treatment response and the grade (1-3) of the immune-related side effect adverse events in the irAE cohort presented as a percentage (\%).

$C R=$ complete response; $P D=$ partial response; $P R=$ progression of disease; $S D=$ stable disease

irAE cohort = patients with metastatic melanoma who developed immune-related adverse events during immunotherapy: NirAE cohort = patients with metastatic melanoma who did not develop immune-related adverse events during immunotherapy

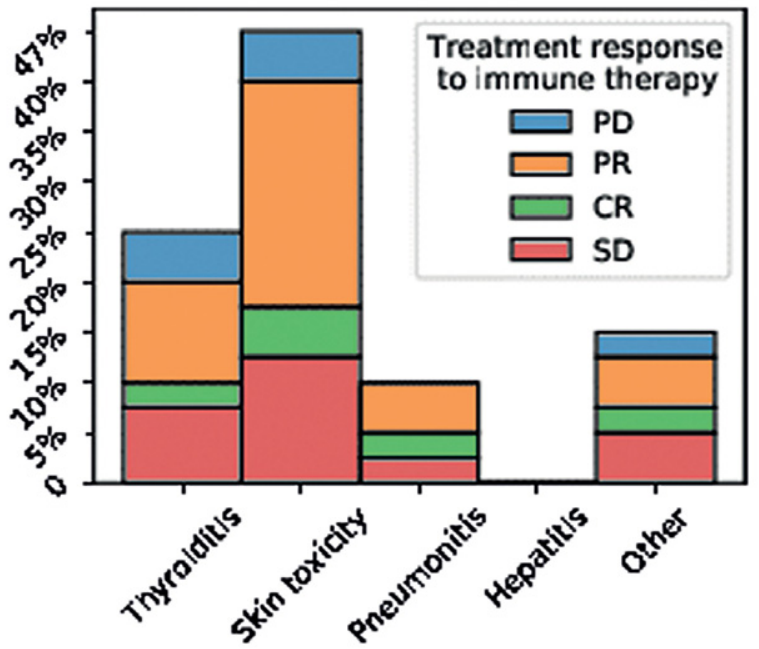

FIGURE 5. CORRELATION between the treatment response and the type of immune-related adverse events in the irAE cohort presented as percentages (\%).

$\mathrm{CR}=$ complete response $\mathrm{PD}=$ partial response; $\mathrm{PR}=$ progression of disease; $S D$ = stable disease

the irAE cohort had progressive disease. The data are presented in Figure 3.

irAE cohort $=$ patients with metastatic melanoma who developed immune-related adverse events during immunotherapy; NirAE cohort = patients with metastatic melanoma who did not develop immune-related adverse events during immunotherapy

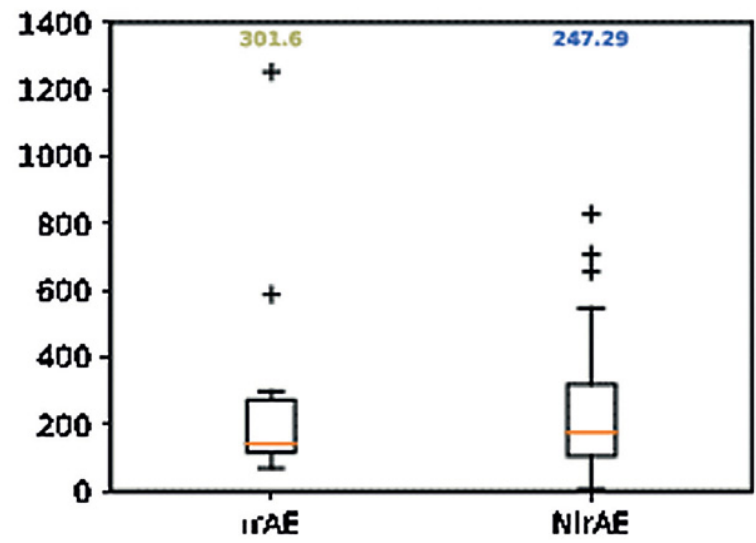

FIGURE 6. Progression-free survival difference in patients with metastatic melanoma between the two cohorts, cohort with immune-related adverse events (irAEs) and cohort with no immune-related adverse events (NirAEs), presented in days. The orange line indicates the median, while the patients who belong to the fourth quartile are represented with plus signs ("+").

irAE cohort = patients with metastatic melanoma who developed immune-related adverse events during immunotherapy; NirAE cohort = patients with metastatic melanoma who did not develop immunerelated adverse events during immunotherapy

Our data show that no patient who developed severe immune-related adverse events (grade 3 ), had progressive disease, as presented in Figure 4 below.

Grade 4 and 5 immune-related adverse events were not present. irAE cohort: patients with metastatic melanoma who developed immune-related adverse events during immunotherapy. 


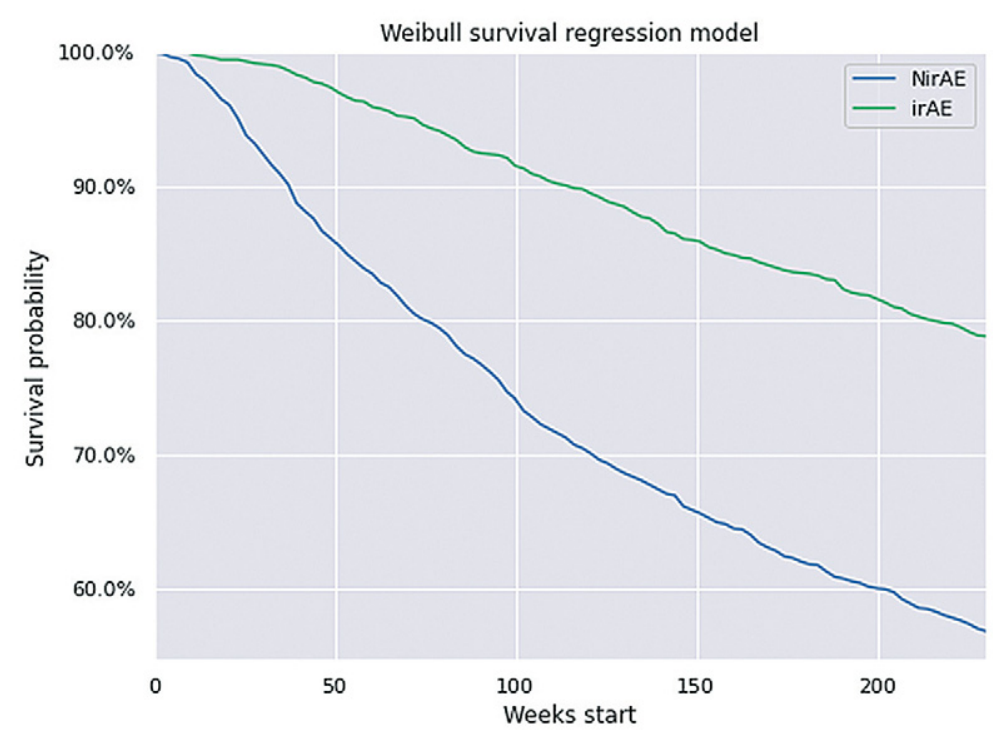

FIGURE 7. Difference in progression-free survival between the irAE and NirAE cohorts, with a significant increase in the survival probability from less than $60 \%$ for the NirAE cohort to almost $80 \%$ for the irAE cohort.

irAE cohort = patients with metastatic melanoma who developed immune-related adverse events during immunotherapy; NirAE cohort = patients with metastatic melanoma who did not develop immune-related adverse events during immunotherapy

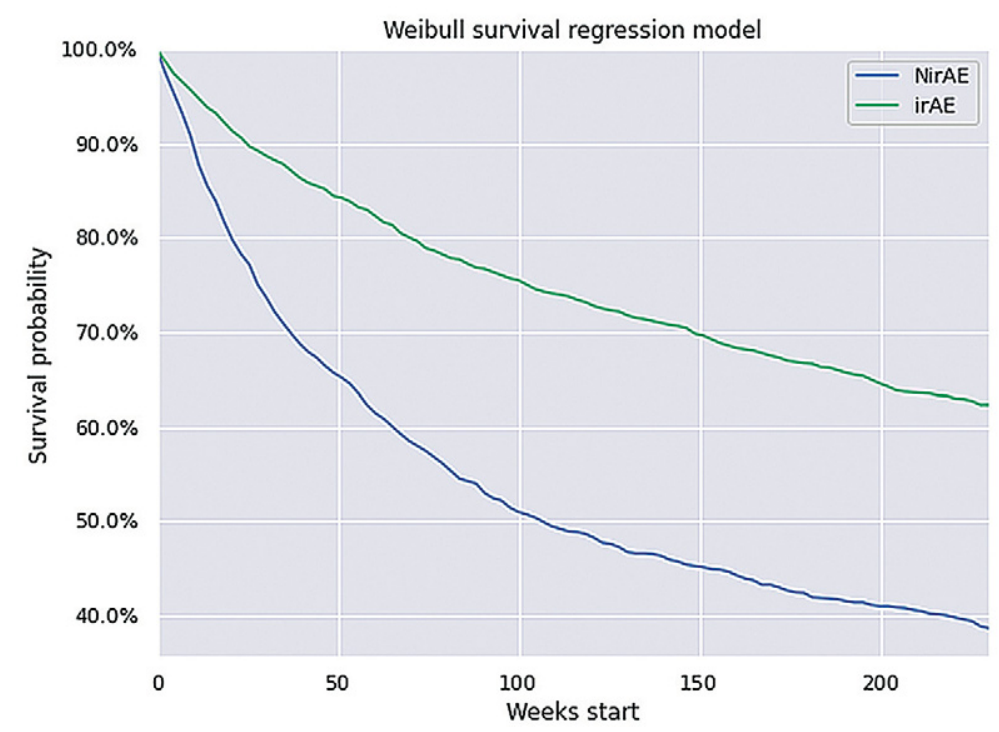

FIGURE 8. Difference in progression-free survival between the irAE and NirAE cohorts with increased LDH, with a significant increase in the survival probability from less than $40 \%$ for the NirAE cohort to more than $60 \%$ for the irAE cohort.

irAE cohor = patients with metastatic melanoma who developed immune-related adverse events during immunotherapy; NirAE cohort = patients with metastatic melanoma who did not develop immune-related adverse events during immunotherapy
No patient who developed immune-related pneumonitis had disease progression, as shown in Figure 5.

Grade 4 and 5 immune: related adverse events were not present; irAE cohort: patients with metastatic melanoma who developed immune-related adverse events during immunotherapy.

Finally, the time to progression of the disease in the cohort (Figure 6) that experienced immunerelated adverse events was significantly longer than the time to progression in the cohort that did not experience immune-related adverse events ( $p$ $=0.001)$. There was no significant difference between the time of progression and the severity of immune-related adverse events.

To investigate the differences in progressionfree survival between the irAE cohort and NirAE cohort, we used survival analysis, which showed a significant increase in the survival probability from less than $60 \%$ for the NirAE cohort to almost $80 \%$ for the irAE cohort (Figure 7). This supports our study hypothesis that patients with immune-related adverse events due to immunotherapy treatment have better treatment outcomes (Figure 7).

A Cox proportional hazards model with covariates time to progression and AE was used for survival analysis. The hazard rate was assumed to be a Weibull distribution. The posterior survival probabilities were obtained through Monte Carlo simulation implemented in Python with the pymc3 package.

Furthermore, the irAE cohort with elevated LDH had better PFS with a $60 \%$ survival probability than the $40 \%$ survival probability for the NirAE cohort with elevated LDH (Figure 8). The same trend was present for the subgroup of patients with irAE stage $\mathrm{M} 1 \mathrm{a} / \mathrm{b}$ melanoma with a survival probability higher than $80 \%$ (Figure 9 ). For the cohort of patients with irAE stage M1c/d melanoma, the results were reversed, showing lower survival probability in comparison with the subgroup of NirAE patients with stage M1c/d melanoma. The survival probability for irAE stage M1c/d patients and NirAE stage M1c/d patients was 50\% and 70\%, respectively (Figure 10).

A Cox proportional hazards model with covariates time to progression and AE was used for survival analysis. The hazard rate was assumed to be a Weibull distribution. The posterior survival probabilities were obtained through Monte Carlo simulation implemented in Python with the pymc3 package.

Cox proportional hazards model with covariates time to progress and AE were used for sur- 
vival analysis. The hazard rate was assumed to be a Weibull distribution. The posterior survival probabilities were obtained through Monte Carlo simulation implemented in Python with pymc3 package.

\section{Discussion}

The main goal of the oncological treatment for metastatic melanoma is progression-free survival while obtaining good quality of life with as few adverse events as possible. Usually, the treatment of adverse events results in treatment delays, decreases quality of life and, consequently, results in loss of disease control and disease progression.

The introduction of immunotherapy in the treatment of metastatic melanoma has improved the prognosis of this disease, prolonging the survival time from less than a year to more than three years..$^{1-3}$ Additionally, recent data show evidence that immunotherapy is much more tolerable, with fewer adverse events than chemotherapy. A metaanalysis of 3450 patients suffering from non-small lung carcinoma and melanoma who were treated with the PD1 inhibitors nivolumab and pembrolizumab and the PDL1 inhibitor atezolizumab showed that compared to chemotherapy, the aforementioned drugs had a significantly lower risk of any all- and high-grade adverse events (fatigue, sensory neuropathy, diarrhoea, haematologic toxicities, anorexia, nausea, and constipation) and consequently a lower rate of treatment discontinuation. ${ }^{10}$

For more than a decade, it has been known that malignant melanoma has a unique immunogenic nature, and the presence of vitiligo in melanoma patients seems to improve the prognosis of melanoma in animals and humans, presenting effective strategy for antitumour immunity. ${ }^{15-17}$

Among immunotherapy drugs used in metastatic melanoma treatment, the CTLA4 inhibitor ipilimumab and the PD1 inhibitors pembrolizum$\mathrm{ab}$ and nivolumab have immune-related adverse events. Ipilimumab is a fully humanized anti-CTLA-4 monoclonal antibody; pembrolizumab and nivolumab are humanized monoclonal anti-programmed cell death-1 (PD-1) antibodies. ${ }^{11-13}$ With the use of CTLA4 inhibitors or anti-PD1 antibodies, also called checkpoint inhibitors, as monotherapy or in combination (nivolumab and ipilimumab), the increased risk of immune-related lung, intestinal, liver, kidney, skin, or endocrine adverse events persists. ${ }^{11-14}$ Due to the severity of the ad-

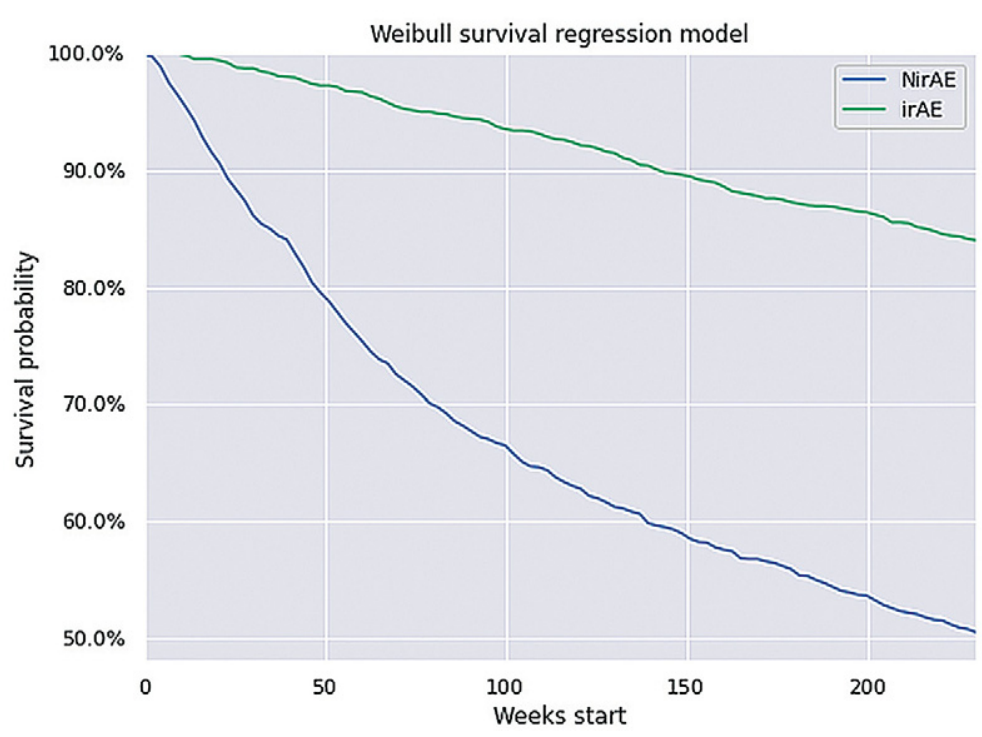

FIGURE 9. Difference in progression free survival between the irAE and NirAE cohort with Mla and Mlb (Mla/b) patients, with a significant increase in the survival probability of approximately $50 \%$ for NirAE cohort to more than $80 \%$ for irAE cohort.

irAE cohort = patients with metastatic melanoma that developed immune-related adverse events during immunotherapy; $\mathrm{Mla} / \mathrm{b}=$ distant metastasis to skin, soft tissue including muscle and/or nonregional lymph node and lungs; NirAE cohort = patients with metastatic melanoma that did not develop immune-related adverse events during immunotherapy;

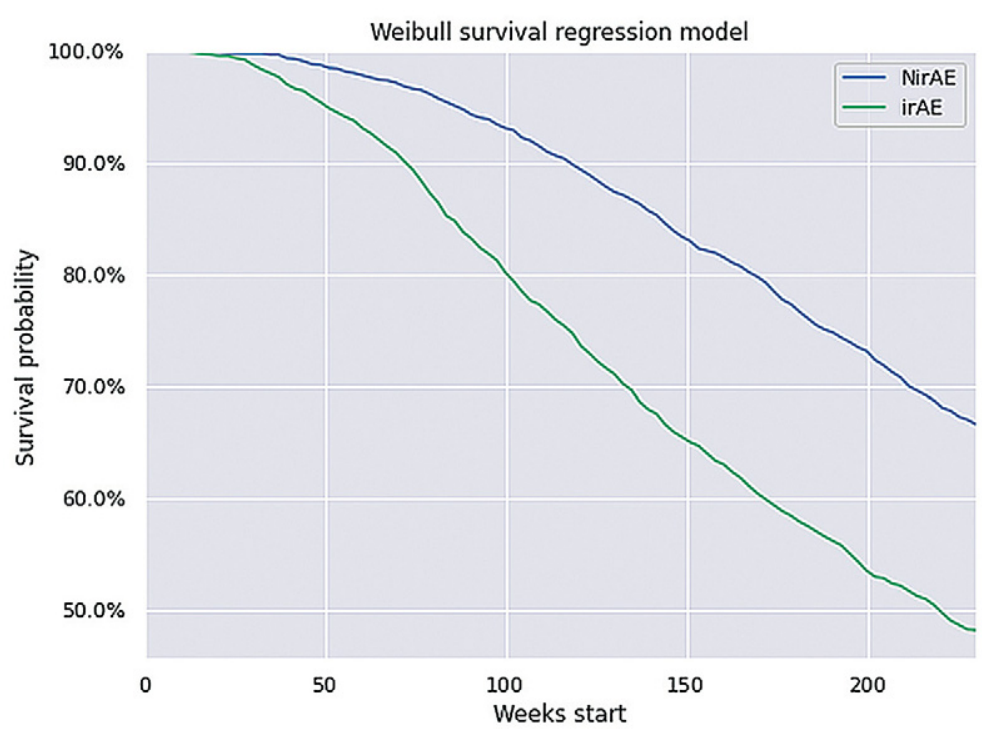

FIGURE 10. Difference in progression free survival between the irAE and NirAE cohort with MlC and Mld (Mlc/d) patients, with a significant increase in the survival probability of almost $70 \%$ for NirAE cohort to less than $50 \%$ for irAE cohort.

irAE cohort = patients with metastatic melanoma that developed immune-related adverse events during immunotherapy; $\mathrm{Mlc} / \mathrm{d}$ = distant metastasis to other visceral sites than lungs and to the central nervous system (CNS); NirAE cohort = patients with metastatic melanoma that did not develop immune-related adverse events during immunotherapy 
verse events caused by immunotherapy treatment, in some cases, discontinuation of the treatment is required. It has been shown that an early discontinuation of immunotherapy due to an adverse event does not negatively affect the long-term survival among these patients. ${ }^{1,3}$

The results of this study show that patients treated with immunotherapy who developed immune-related adverse events had better treatment outcomes than patients without immune-related adverse events. This retrospective study, performed on 99 patients with metastatic melanoma who were treated with immunotherapy, showed that patients with immune-related adverse events had an improved ORR in comparison to the ORR of patients without immune-related adverse events (75\% vs. 37\%). The PFS was significantly longer for the patients with immune-related adverse events, 301.6 days, compared to 247.29 days for patients without immune-related adverse events. Neither the severity nor the type of immune-related adverse events correlated with the ORR or PFS.

The presented data are in line with recent publications reporting a positive correlation between immune-related adverse events and survival. ${ }^{7-9}$ A Dutch prospective study on 147 patients with metastatic melanoma treated with pembrolizumab showed that high-grade toxicity at any time during treatment was associated with higher objective response rate, progression-free survival, and overall survival. ${ }^{7}$ A retrospective study on 144 metastatic melanoma patients treated with pembrolizumab showed similar results, as the development of any irAE (HR, 0.24, P < .001) was significantly associated with longer OS times. ${ }^{8}$

The Cox proportional hazards regression analysis in this study shows a difference in progressionfree survival between the irAE and NirAE cohorts, with a significantly increased survival probability from less than $60 \%$ for the NirAE cohort to almost $80 \%$ for the irAE cohort. Furthermore, the subgroup of patients with irAEs with elevated LDH, before the start of immunotherapy, had better PFS, with a $60 \%$ survival probability compared to the $40 \%$ survival probability for the subgroup of NirAE patients with elevated LDH. The same pattern was observed for the subgroup of patients with irAEs and stage $\mathrm{M} 1 \mathrm{a} / \mathrm{b}$ with a survival probability of greater than $80 \%$. The findings were reversed for the subgroup of patients with irAEs and stage M1c/d melanoma, where the survival probability was lower than that of the subgroups of patients with NirAEs and stage M1c/d mela- noma, with survival probabilities of $50 \%$ and $70 \%$, respectively. There were only a few patients with increased LDH for each M1 stage; hence, we omitted these patients from multivariate analysis.

Elevated LDH is a poor prognostic marker for melanoma patients; however, LDH and immunerelated side effects are widely used for the prognosis of immunotherapy outcomes. ${ }^{18}$ Immunotherapy is effective for melanoma patients with dissemination locations indicating poor prognosis (M1c/d) $)^{19}$; however, there is a lack of data regarding the negative correlation in patients with immune-related side effects. As reported, developing immunerelated adverse events correlates with better treatment outcomes. ${ }^{7-9,18}$ Dissemination of melanoma in visceral organs other than the lungs and CNS, historically, is related to poor prognosis and outcome. The response rate of melanoma patients with brain metastases ranges from $26 \%$ with PD1 inhibitors to $55 \%$ with a combination of CTLA 4 and PD1 inhibitors. ${ }^{20,21}$ The expected time to response is longer, and the risk for hyperprogression in this subgroup of melanoma patients is higher. 22,23 Our data, though represented by a small group of patients, contribute to the possibility of new melanoma entities with worse immunotherapy outcomes-i.e., the subgroup of irAE patients with stage M1c/d disease. Potentially, the small group of patients may lead to bias; hence, a broader retrospective analysis of patients with metastatic melanoma treated with immunotherapy at the Institute of Oncology Ljubljana is planned in the future.

\section{Conclusions}

Our study indicates a positive correlation of the higher autoimmunogenicity caused by immunotherapy in patients with metastatic melanoma with the treatment outcome and thus improves knowledge about immunotherapy. In the present cohort, patients with immune-related adverse events during immunotherapy had better ORRs, OS and PFS than patients with metastatic melanoma without any immune-related adverse events. The Cox proportional hazards regression analysis showed a difference in PFS between the irAE and NirAE cohorts, with a significant increase in the survival probability from less than $60 \%$ for the NirAE cohort to almost $80 \%$ for the irAE cohort even in the presence of elevated LDH. This pattern was not observed for the group of patients with M1c/d disease, stipulating the need for further research. 


\section{Acknowledgement}

We thank Pavle Boškoski for his contribution to the survival regression model analysis. TM, VČM, JO acknowledges funding from the Slovenian Research Agency via program P3-0321. BMB acknowledges funding from the Slovenian Research Agency via program Complex Networks P1-0383.

\section{References}

1. Larkin J, Chiarion-Sileni V, Gonzalez R, Grob JJ, Rutkowski R, Lao CD, et al. Five-year survival with combined nivolumab and ipilimumab in advanced melanoma. N Engl J Med 2019; 381: 1535-46. doi: 10.1056/ NEJMoa1910836

2. Hamid O, Robert C, Daud A, Hodi FS, Hwu WJ, Kefford R, et al. Five-year survival outcomes for patients with advanced melanoma treated with pembrolizumab in KEYNOTE-001. Ann Oncol 2019; 30: 582-8. doi: 10.1093/ annonc/mdz011

3. Robert C, Hwu W-J, Hamid O, Ribas A, Weber JS, Daud Al, et al. Long-term safety of pembrolizumab monotherapy and relationship with clinical outcome: a landmark analysis in patients with advanced melanoma. Eur J Cancer 2021; 144: 182-91. doi: 10.1016/j.ejca.2020.11.010

4. Hocevar M, Strojan P, Ocvirk J, Peric B, Blatnik O, Luzar B, et al. [Recommendation for the treatment of patients with melanoma]. [Slovenian]. Rebersek M, editor. [cited 2020 Oct 15]. Ljubljana: Institute of Oncology Ljubljana; 2019. Available at: https://www.onko-i.si/fileadmin/ onko/datoteke/Strokovna_knjiznica/smernice/Priporocila_za_obravnavo_ bolnikov_z_melanomom_2020.pdf

5. Michielin O, van Akkooi ACJ, Ascierto PA, Dummer R, Keilholz U, on behalf of the ESMO Guidelines Committee. Cutaneous melanoma: ESMO Clinical Practice Guidelines for diagnosis, treatment and follow-up. Ann Oncol 2019; 30: 1884-901. doi: 10.1093/annonc/mdz411

6. NCCN Clinical Practice Guidelines in Oncology, Cutaneous melanoma. Version 4.2020. [cited 2020 Oct 14]. Available at: https://www.nccn.org/ professionals/physician_gls/pdf/cutaneous_melanoma.pdf

7. Bisschop $C$, Wind $T$, Blank CU, Koornstra RHT, Kapiteijn E, Van den Eertwegh AJM, et al. Association between pembrolizumab-related adverse events and treatment outcome in advanced melanoma: results from the Dutch Expanded Access Program. J Immunother 2019; 42: 208-14. doi: 10.1097/CJl.0000000000000271

8. Suo AE, Chan Y, Cheung WY, Monzon JG, Smylie M, Walker JWT, et al. Pembrolizumab-induced immune related adverse events and survival outcomes in advanced melanoma. [abstract]. J Clin Oncol 2018; 36 (15 Suppl): e21577. doi: 10.1200/JCO.2018.36.15 suppl.e21577

9. Hussaini S, Chehade R, Boldt RG, Raphael J, Blanchette P, Maleki Vareki S, et al. Association between immune-related side effects and efficacy and benefit of immune checkpoint inhibitors - a systematic review and meta-analysis. Cancer Treat Rev 2021; 92: 102134. doi: 10.1016/j.ctrv.2020.102134

10. Nishijima TF, Shachar SS, Nyrop KA, Muss HB. Safety and tolerability of PD-1/ PD-L1 inhibitors compared with chemotherapy in patients with advanced cancer: a meta-analysis. Oncologist 2017; 22: 470-9. doi: 10.1634/theoncologist.2016-0419

11. EMA. Annex I. Summary of product characteristics. Ipilimumab (Yervoy) product information. [cited 2020 Oct 13]. Available at: https://www.ema. europa.eu/en/documents/product-information/yervoy-epar-product-information_en.pdf

12. EMA. Annex I. Summary of product characteristics. Pembrolizumab (Keytruda) product information: [cited 2020 Oct 12]. https://www.ema. europa.eu/en/documents/product-information/keytruda-epar-productinformation_en.pdf

13. EMA. Annex I. Summary of product characteristics. Nivolumab (Opdivo) product information: [cited 2020 Oct 11]. https://www.ema.europa.eu/en/ documents/product-information/opdivo-epar-product-information_sl.pdf
14. Hribernik N, Boc M, Ocvirk J, Knez-Arbeiter J, Mesti T, Ignjatovic M, et al. Retrospective analysis of treatment-naive Slovenian patients with metastatic melanoma treated with pembrolizumab - real-world experience. Radiol Oncol 2020; 54: 119-27. doi: 10.2478/raon-2020-0003

15. Bystryn JC. Serum antibodies in vitiligo patients. Clin Dermatol 1989; 7: 13645; doi: 10.1016/0738-081x(89)90063-1

16. Oyarbide-Valencia K, van den Boorn JG, Denman CJ, Li M, Carlson JM, Hernandez C, et al. Therapeutic implications of autoimmune vitiligo T cells. Autoimmun Rev 2006; 5: 486-92. doi: 10.1016/j.autrev.2006.03.012

17. Hua C, Boussemart L, Mateus C, Routier E, Boutros C, Cazenave H, et al. Association of vitiligo with tumor response in patients with metastatic melanoma treated with pembrolizumab. JAMA Dermato/ 2016; 152: 45-51. doi: 10.1001/jamadermatol.2015.2707

18. Dick J, Lang N, Slynko A, Kopp-Schneider A, Schulz C, DimitrakopoulouStrauss $A$, et al. Use of $\mathrm{LDH}$ and autoimmune side effects to predict response to ipilimumab treatment. Immunotherapy 2016, 8: 1033-44. doi: 10.2217/imt-2016-0083

19. Olson D, Luke JJ, Poklepovic AS, Bajaj M, Higgs E, Carll TC, et al. Significant antitumor activity for low-dose ipilimumab (IPI) with pembrolizumab (PEMBRO) immediately following progression on PD1 Ab in melanoma (MEL) in a phase II trial. [abstract]. J Clin Oncol 2020; 38 (Suppl 15): 10004. doi: 10.1200/JCO.2020.38.15_suppl.10004

20. Kluger HM, Chiang V, Mahajan A, Zito CR, Sznol M, Tran T, et al. Long-term survival of patients with melanoma with active brain metastases treated with pembrolizumab on a phase II trial. J Clin Oncol 2019; 37: 52-60. doi: 10.1200/JCO.18.00204.

21. Long GV, Atkinson V, Lo S, Sandhu S, Guminski AD, Brown MP, et al. Combination nivolumab and ipilimumab or nivolumab alone in melanoma brain metastases: a multicentre randomised phase 2 study. Lancet Oncol 2018; 19: 672-81. doi: 10.1016/S1470-2045(18)30139-6

22. Tawbi HA, Forsyth PA, Algazi A, Hamid O, Hodi FS, Moschos SJ, et al. Combined nivolumab and ipilimumab in melanoma metastatic to the brain N Engl J Med 2018; 379: 722-30. doi: 10.1056/NEJMoa1805453 